\title{
Música, Mujeres y Disidencias: Limitaciones estructurales y principales obstáculos en la construcción de la música como profesión en Uruguay
}

\author{
Música, Mulheres e Dissidência: Limitações estruturais e principais \\ obstáculos na construção da música como profissão no Uruguai
}

\author{
Music, Women and Dissidence: Structural limitations and main obstacles \\ in the construction of music as a profession in Uruguay
}

Sol Scavino ${ }^{1}$ y Paula Simonetti ${ }^{2}$

\begin{abstract}
Resumo
Neste artigo analisamos as limitações estruturais e os principais obstáculos enfrentados pelas mulheres e outras identidades de gênero no desenvolvimento da profissão musical no Uruguai. Fazemos isso a partir da análise de fontes secundárias (Censos Populacionais 1985 e 1996) e Pesquisas Domiciliares Contínuas (2015-2018), representativas da população uruguaia. A partir destas, procedemos a uma caracterização quantitativa da situação laboral de mulheres e homens cuja principal ocupação é o trabalho artístico, em particular a música. As desigualdades de gênero na música e os principais problemas que implicam para o desenvolvimento do trabalho artístico são abordados a partir da análise de uma amostra não probabilística, que emerge dos dados de um formulário autoaplicável por inscritas no I Encuentro de Mujeres y Disidencias de la Música Uruguay (MasMúsicas, 2019). O estudo analisa as características específicas do mercado de trabalho artístico, suas condições estruturais e simbólicas, e sintetiza as desigualdades de gênero no trabalho cultural na perspectiva das mulheres e outras identidades de gênero que atuam no campo da música.
\end{abstract}

Palavras-chave: condições de trabalho; gênero; música; trabalho artístico

\section{Resumen}

En este artículo analizamos las limitaciones estructurales y los principales obstáculos que se presentan para mujeres y disidencias en el desarrollo de la profesión musical en Uruguay. Lo hacemos a partir del análisis de fuentes secundarias (Censos de Población 1985 y 1996) y Encuestas continuas de Hogares (2015-2018), representativas de la población uruguaya. A partir de los mismos realizamos una caracterización cuantitativa de la situación laboral de mujeres y varones cuya principal ocupación sea el trabajo artístico, particularmente, la música. Las desigualdades de género en la música y las principales problemáticas que implican para el desarrollo del trabajo artístico se abordan a partir del análisis de una muestra no probabilística, que emerge de los datos de un formulario auto administrado por las inscriptas al Primer Encuentro de Mujeres y Disidencias de la Música Uruguay (MasMúsicas, 2019). El trabajo analiza las características específicas del mercado del trabajo artístico, sus condicionamientos estructurales y simbólicos, y sintetiza las desigualdades de género en el trabajo cultural desde la perspectiva de las mujeres y disidencias músicas.

\footnotetext{
${ }^{1}$ Sol Scavino, Magíster en Sociología con especialización en Género. Candidata a Doctora en Sociología por la Universidad de la República. Investigadora y profesora Asistente del Departamento de Sociología, Facultad de Ciencias Sociales, Udelar. Email: sol.scavino@cienciassociales.edu.uy

${ }^{2}$ Paula Simonetti, Magíster en Sociología de la Cultura, Doctorando en Sociología (IDAES-UNSAMCONICET) Email: psimonetti@unsam.edu.ar
} 
Palabras clave: condiciones laborales; disidencias; género; música; trabajo artístico

\begin{abstract}
In this paper, we analyze the structural limitations and the main obstacles faced by women and other gender identities in the development of the musical profession in Uruguay. We do so from the analysis of secondary sources (Population Censuses 1985 and 1996) and Continuous Household Surveys (2015-2018), representative of the Uruguayan population. Based on these, we carry out a quantitative characterization of the employment situation of women and men whose main occupation is artistic work, particularly music. Gender inequalities in music and the main problems they imply for the development of artistic work addressed from the analysis of a non-probabilistic sample, which emerges from the data of a self-administered form by those registered in the Primer Encuentro de Mujeres y Disidencias de la Música Uruguay (MasMúsicas, 2019). The study analyzes the specific characteristics of the artistic work market, its structural and symbolic conditions, and synthesizes gender inequalities in cultural work from the perspective of women and other gender identities that work in the field of music.
\end{abstract}

Key words: working conditions; gender; music; artistic work

\title{
1. Introducción
}

En el presente artículo analizamos las limitaciones estructurales y los principales obstáculos que se presentan para mujeres y disidencias en el desarrollo de la profesión musical en Uruguay. Se busca generar un diálogo a partir de las reflexiones sobre las características específicas del mercado del trabajo artístico, sus condicionamientos estructurales y simbólicos y la especificidad que la perspectiva de género puede aportar al análisis del trabajo en cultura. El texto está estructurado de la siguiente manera: presenta, en primer lugar, un panorama de las características generales que estructuran los mercados de trabajo artístico, incorporando estudios internacionales y regionales; luego, una discusión sobre elementos simbólicos e identitarios que tensionan y pueden obstruir la construcción de la identidad laboral de lxs trabajadores de la cultura; a continuación presentamos antecedentes regionales acerca de la dimensión de género en las prácticas y trabajos culturales, y se profundiza específicamente en ciertos movimientos sociales abocados a paliar las desigualdades de género en la escena musical. Los siguientes apartados se dedican in extenso al estudio de los datos hallados para el caso de las mujeres y disidencias en la música uruguaya. Estos surgen del análisis de fuentes secundarias de carácter representativo a nivel nacional como los Censos de Población, Vivienda y Hogares (1985 y 1996), para los cuales se cuenta con información sobre la ocupación principal de las personas, entre otras variables sociodemográficas de interés. Puesto que el Censo de 2011 no cuenta con una desagregación a cuatro dígitos de la ocupación principal de las personas, a fin de conocer cómo son las situaciones ocupacionales de varones y mujeres en la música, se procesaron datos de las 
Encuestas Continuas de Hogares (2015 a 2018), elaboradas por el Instituto Nacional de Estadística de Uruguay. Las mismas representan a la población total (exceptuando ruralidad dispersa) y relevan aspectos sociodemográficos, educativos, laborales y de ingresos. Al estudio de carácter representativo, se le suma la caracterización de las mujeres y disidencias en la música, a partir del análisis de los datos que no obedecen a un muestreo probabilístico, de un formulario autoadministrado, de las personas inscriptas al Primer Encuentro de Mujeres y Disidencias en la Música Uruguaya ${ }^{3}$ (PEMDMU-MásMúsicas, 2019). Se caracterizó al colectivo en relación a los datos representativos a nivel nacional para dar cuenta de las particularidades del grupo de mujeres y disidencias. Por último, ofrecemos algunas reflexiones a modo de balance final.

\section{El mundo del trabajo cultural y artístico: algunos rasgos estructurales}

Numerosos estudios a nivel internacional evidencian características comunes entre lxs artistas en tanto grupo ocupacional. Se destaca que son en promedio más jóvenes que la fuerza laboral en general, tienen altos niveles de educación, muestran tasas altas de autoempleo, de desempleo y presentan varias formas de subempleo y de multiempleo (OLIVERA GUADARRAMA et. al., 2012; MENGER, 1999). Asimismo, suelen percibir salarios menores que 1xs trabajadores en sus categorías profesionales de referencia, profesionales, técnicos y afines, "cuyos miembros tienen características de capital humano comparables (educación, capacitación y edad) y tienen mayor variabilidad y calidad de ingresos" (MENGER, 1999, p.545, traducción nuestra). Algunos análisis sugieren una relación directa entre la inestabilidad, la precariedad laboral y la multiactividad en estas ocupaciones (OLIVERA GUADARRAMA et. al., 2012). No obstante, diversas investigaciones alertan sobre una variedad de dificultades metodológicas para el abordaje del problema de la precariedad laboral, y es preciso señalar que no se reduce "a una división entre empleos precarios y no precarios, sino que debe entenderse como un continuum, expresado por grados de precariedad y vulnerabilidad, que puede afectar a empleos aparentemente seguros" (ESOPE, 2005, p.48). Para posibilitar su observación empírica, Esope distingue cuatro dimensiones: (1) temporal: refiere al grado de certeza acerca de la continuidad del empleo; (2) organizacional: refiere al control individual y colectivo de lxs trabajadores sobre el trabajo; (3) económica: pago suficiente y progresión salarial; y (4)

\footnotetext{
${ }^{3}$ La organización Más Músicas Uruguay ha compartido con nosotras los datos del PEMDMU para la elaboración del presente artículo en un acto de generosidad que agradecemos y valoramos como práctica en la co-construcción de conocimiento entre organizaciones sociales y académicas.
} 
social: la protección legal contra despidos injustos, discriminación en el trabajo, protección social (beneficios de salud, seguridad social, desempleo, accidentes, etc.).

Por otro lado, es posible afirmar que en el mundo de la producción cultural, encontramos una frontera borrosa entre el trabajo remunerado y no remunerado, así como entre profesionales y amateurs. De hecho, tal como apuntan Hesmondhalgh y Baker (2011) no es infrecuente que el trabajo no remunerado sea la base de una reputación que permite a las personas volverse profesionales (2011, p.17).

De manera coincidente con otros análisis internacionales, para el caso de la profesión musical en México, Guadarrama et al. (2012) señalan que la característica más sobresaliente de los patrones de empleo de músicos profesionales es la multiactividad, se trata de un sector en que los individuos se movilizan constantemente de un empleo a otro, por períodos de tiempo breves, con arreglos entre distintos tipos de contratos.

Esta conclusión también es respaldada por algunos foros internacionales como la Federación Internacional de Músicos (1997) y la Organización de las Naciones Unidas para la Educación, la Ciencia y la Cultura (UNESCO), cuando comparan la situación laboral de los músicos de distintos países con las convenciones de la Organización Internacional del Trabajo (OIT) y las recomendaciones más específicas sobre el trabajo artístico de la propia UNESCO (1997).

En cuanto a la OIT, se ha ubicado el trabajo artístico como trabajo atípico, donde prevalece la figura del trabajador independiente, por cuenta propia, o inmerso en la economía informal (OIT, 2004). En un informe del 2014, donde se analizan las tendencias laborales en las industrias culturales y de medios, señalan que se incrementa el empleo cultural pero bajo la modalidad del contrato atípico. A su vez, se visualiza la incrementación de la formación permanente pero esto no se traduce en un aumento de la demanda laboral, y se identifica entre los jóvenes y las mujeres a los sectores más afectados por la percepción de menores ingresos y peores condiciones laborales. Por su parte, el estudio chileno El Escenario del Trabajador Cultural en Chile (2014) apunta asimismo a la precariedad en que se desarrolla el trabajo artístico cultural en dicho país, destacando la desprotección social de lxs trabajadores, la prevalencia del trabajo informal, independiente, sin contrato y sin previsión social. También encuentran que la actividad artística se combina con actividades no culturales, acentuándose la diversificación del empleo y la multiactividad (2014, p.219).

\section{Tensiones simbólicas e identitarias en el desarrollo profesional artístico}


$\mathrm{Si}$ bien es innegable que las condiciones laborales anteriormente descritas pueden inscribirse en transformaciones más amplias que atraviesa el mercado de trabajo, caracterizado cada vez más por su condición inestable, flexibilizada, insegura y precaria, también es cierto que existen condiciones específicas, donde elementos como la inestabilidad laboral o la precariedad en las contrataciones dialogan con ciertas oposiciones de sentidos que permean los ámbitos mencionados, tensionándolos: arte/trabajo, creatividad/seguridad, incertidumbre/estabilidad, interés/desinterés, placer/utilidad, por ejemplo.

Centrada en el estudio de las artes escénicas en Buenos Aires, Mauro (2018) identifica una imbricación entre modos de producción que suponen precarización laboral o incluso la gratuidad del trabajo artístico, con la construcción identitaria que realizan de sí mismos lxs artistas. La idea de que el trabajo artístico o cultural no debería estar subordinado a fines económicos se encuentra subyacente en nociones como la vocación, atribuida socialmente a los artistas (aunque no exclusivamente), o a la idea de que "el placer que reporta el ejercicio de determinada práctica sustituiría la falta de remuneración (lo cual constituye también una condena implícita al placer)" (MAURO, 2018, p.116). Muchas de estas representaciones subyacen a las políticas culturales y científicas. De este modo, apunta a la necesidad de fortalecer la estructura identitaria y la autopercepción de lxs artistas en tanto trabajadores, puesto que una de las dificultades básicas para la exigencia de políticas culturales que protejan y regulen estos trabajos, radica en comenzar a percibir la gratuidad y la precariedad del trabajo artístico como un problema (MAURO, 2018, p.256). En una línea similar, Infantino (2011) muestra que, históricamente, se han generado sentidos valorativos contrarios entre las esferas del arte y el trabajo, donde el arte se coloca en un plano de autonomía y diferencia, se le atribuye la búsqueda de la autenticidad, la verdad, la belleza, enfrentado a valores asignados al mundo de la economía (la búsqueda racional de la ganancia o el instrumentalismo ilimitado) (INFANTINO, 2011, p.141). Estas problemáticas se vinculan con el análisis de Bourdieu (1997) sobre la economía de los bienes simbólicos, en donde pone en relación el mundo doméstico, el artístico y el religioso para ilustrar que en ellos se crean condiciones objetivas para que los agentes sociales tengan interés en el "desinterés", aunque pueda resultar paradójico (1997, p.160). En estos mundos, el autor encuentra una lógica en donde existe una represión, o censura, del interés económico —en sentido restringido-, señalando que la "verdad económica" (el precio) se oculta o se deja sin precisar. La economía de los bienes simbólicos se transforma, así, en una economía de lo difuso e indeterminado. 
Por su parte, Lorey (2006) apunta que al interior del grupo de productores culturales se impone la creencia de que se han elegido las propias condiciones vitales y laborales, y que estas se realizan de manera relativamente autónoma y libre. Así, se propone mostrar que las ideas de autonomía y libertad están constitutivamente conectadas con los modos hegemónicos de subjetivación en las sociedades capitalistas occidentales.

Las tensiones del mundo profesional de la música han sido abordados en antecedentes que destacan las contradicciones entre el trabajo por vocación y el trabajo por supervivencia, el trabajo creativo o "por amor al arte", contrapuesto con emprendimientos con beneficios económicos (BECK, 2003; CAVES, 2002; COULSON, 2012). Estos también señalan como fuentes de tensión la organización del trabajo por proyecto y la intermitencia.

\section{Género y trabajo cultural}

Las características estructurales y simbólicas del trabajo artístico también están atravesadas por las desigualdades de género, que afectan todas las formas de trabajo. La participación de las mujeres y disidencias (MyD) en el ámbito de la política, el trabajo remunerado, las instituciones tomadoras de decisiones en cultura, es escasa. Comprender este fenómeno requiere de un ejercicio de vigilancia epistemológica sobre los prejuicios que señalan que las MyD no ocupan lugares de visibilidad en la música porque no quieren, no están aptas o tienen otros intereses (vinculados a lo reproductivo y doméstico). El arraigo cultural que conlleva la asociación del trabajo de cuidados con las mujeres, se evidencia en las representaciones sociales, estereotipos y producciones de subjetividades de género, que sostienen y acompañan una aún persistente división sexual del trabajo (DST) (BIANCHI et. al., 2000; DAVIS et. al., 2007; SEVILLA-SANZ et. al., 2010; TREAS yDROBNIC, 2010; SAYER, 2010; BATTHYÁNY, GENTA y PERROTA, 2012; BATTHYÁNY y SCAVINO, 2017; OIT, 2018). La DST plantea, grosso modo, que a las mujeres se les asigna el trabajo doméstico y de cuidados como ámbito de desempeño (ámbito privado) y a los varones la esfera política, mercantil y cultural (ámbito público) ${ }^{4}$. Estos espacios son valorados de manera diferente (simbólica y económicamente), identificándose en la DST un principio de separación (hay trabajos "de hombres" y "de mujeres") y un principio de jerarquía que establece que el trabajo realizado por los varones "vale más" que el trabajo realizado por las mujeres (KEROGAT, 2003, p.845).

\footnotetext{
${ }^{4} \mathrm{Si}$ bien este esquema ha sido criticado por reduccionista de las múltiples realidades del trabajo y por plantear una dicotomía público/privado, varón/mujer cuestionable (HARAWAY, 1995; BRAIDOTTI, 1991; BUTLER, 2001), constituye una herramienta heurística para comprender las relaciones de género. Particularmente, si se considera que el tiempo es un recurso escaso, sobre el que la capacidad de tomar decisiones en primera persona puede verse condicionada por lo que se espera que los sujetos hagan conforme su edad, su clase, su ascendencia étnico-racial, su género.
} 
La desvalorización del trabajo realizado por las mujeres ha sido problematizada, buscando identificar y desmontar los mecanismos que mantienen oculto el carácter productivo y la complejidad que caracteriza al trabajo de cuidados y doméstico. A su vez, los estudios del mercado laboral muestran que los sectores feminizados pagan menos que los masculinizados, contribuyendo a la conformación de una brecha salarial de género. También se subraya que la ausencia de corresponsabilidad en los cuidados ha generado altos costos para las mujeres y disidencias en sus trayectorias laborales y estudiantiles y el uso del tiempo en sus vidas cotidianas (KAPLAN, 1987; LONGO, 2009; CARRASCO, BORDERÍAS, y TORNS, 2011; TORNS, 2008; TORNS et. al., 2012; BATTHYÁNY, 2015).

Si bien aún son escasos los análisis que retomen la perspectiva de género para analizar las dinámicas de la producción cultural, es posible destacar algunos antecedentes. Por ejemplo, el informe argentino Mujeres en la cultura: notas para el análisis del acceso y la participación cultural en el consumo y el mercado de trabajo (MINISTERIO de CULTURA, 2018), ${ }^{5}$ entre otros hallazgos, señala que es en los espacios comunitarios donde se observa una participación más pareja entre mujeres y varones, sin embargo, existe una diferencia significativa en tanto que los últimos ocupan más cargos jerárquicos que las mujeres, de esta manera, en figuras como dirigente, organizador o coordinador, la proporción de varones duplica a la de las mujeres ${ }^{6}$. El informe señala, asimismo, que en el ámbito de las industrias culturales la brecha de ingresos entre varones y mujeres asciende al $28 \%$ en favor de los primeros, situación bastante equiparable a la que se constata en el mercado laboral no cultural (aproximadamente del 23\%).

Para el caso español, Guirao (2019) muestra que, a pesar de que se observa una tendencia a la feminización de los consumos y producciones culturales, no se ha producido el reconocimiento social en igualdad de estos bienes. Basándose en datos de la Encuesta de Población Activa en el Ámbito Cultural, la investigadora señala que las mujeres ocupan los empleos relacionados con las industrias culturales tradicionales: bibliotecas, archivos, museos, edición de libros o periódicos, un 56,1\% de mujeres, 43,9\% hombres. En cambio, los varones ocupan empleos más cercanos a las nuevas industrias creativas. Por su parte, señala

\footnotetext{
${ }^{5}$ En este estudio para el análisis de la participación de mujeres en el mercado del trabajo cultural se siguió la propuesta de UNESCO presente en el documento Marco de Estadísticas Culturales (2009). El nivel de empleo en industrias y profesiones culturales se realizó utilizando la Encuesta Permanente de Hogares realizada por el Indec.

${ }^{6}$ Por otro lado, en los motivos que las personas aducen acerca de por qué no concurren a determinados eventos culturales, observan que el motivo de no asistencia "por trabajo": de cada 10 personas que argumentaron no asistir a recitales por motivos laborales 9 son varones. En contrapartida, 8 de cada 10 personas que respondieron que no asisten a recitales por "motivos familiares, como tener hijos pequeños", son mujeres.
} 
que si bien se constata que las mujeres escriben, pintan y dibujan más que los hombres, cuando estos datos se cruzan con los registros de propiedad intelectual en concepto de autor, se visualiza que los varones registran sustancialmente más que las mujeres sus obras.

\subsection{Movimientos sociales por las desigualdades de género en la música}

Las desigualdades mencionadas anteriormente se dan en un marco latinoamericano en donde el movimiento social feminista se ha expandido, buscando colocar debates en torno a los derechos de salud sexual y reproductiva, a la participación equitativa en ámbitos como la cultura, el trabajo, la política, en el acceso al disfrute y el tiempo libre en las agendas públicas. El surgimiento de múltiples organizaciones de mujeres y disidencias en el arte, comenzó a visibilizar la masculinización de las artes como una problemática y a enunciar las dificultades que existen para participar y acceder a financiamiento para desarrollar su trabajo. En el caso uruguayo, principalmente en Montevideo y en el ámbito de la música, la presencia de Ronda de Mujeres (2002-a la fecha), que congrega mujeres DJ's (IM-RMD, 2018), el florecimiento de las críticas feministas al carnaval y las organizaciones de mujeres murguistas (2017-a la fecha) ${ }^{7}$, así como el trabajo desarrollado por el Departamento de Cultura de la Intendencia de Montevideo (2015-2020), a cargo de Mariana Percovich, que dio viabilidad a los compromisos que la IM asumió en el 3er Plan de Igualdad de Oportunidades entre varones y mujeres para el período 2016-2020, son algunos ejemplos del surgimiento de una masa crítica que comienza a poner el foco en las desigualdades de género en la cultura. Paralelamente, en Argentina se aprobó recientemente la Ley $N^{\circ} 25.539$ referida al "Cupo femenino y acceso de artistas mujeres a eventos musicales", que instala un antecedente sobre el cual discutir cuotas en espectáculos como mecanismo para garantizar la participación de los colectivos subrrepresentados. En Uruguay, la discusión acerca de cómo garantizar la participación y visibilización de las mujeres y disidencias mediante políticas culturales es incipiente.

\footnotetext{
${ }^{7}$ Entre 2017 y 2018, las mujeres murguistas organizadas y las murgas feministas desarrollaron el Encuentro Nacional de Mujeres Murguistas, en 2019 llevaron a cabo el Encuentro de Murgas de Mujeres y Mujeres Murguistas y en 2020, convocaron al Encuentro de Murgas Feministas. En el ámbito del carnaval, la murga (una de las expresiones más relevantes de la cultura e identidad, principalmente montevideana) está fuertemente masculinizada. A su vez, los textos de las murgas suelen ser fuertemente discriminatorios con las mujeres y disidencias y tradicionales en relación a los roles de género. Los datos oficiales sobre la participación de las mujeres en Carnaval son escasos y tienen apertura por la variable sexo solo desde 2017. Datos solicitados a la Secretaría de Gerencia de Festejos y Espectáculos del Departamento de Cultura de la Intendencia de Montevideo (2018), permitieron evidenciar una fuerte segregación en la composición de la participación en distintos ámbitos del carnaval por sexo. En el Concurso de Carnaval oficial, se destacó que solo 23,6\% de las que participan son mujeres. Una proporción similar se observa en el caso de Murga Joven (23,9\%). En el Carnaval fuera de concurso, solo $16,3 \%$ son mujeres.
} 
A continuación, nos centraremos en identificar las características del trabajo de las MyD en la música, y las principales problemáticas experimentadas por ellas. La identificación de estos aspectos se enmarca en las acciones de MásMúsicas Uruguay, organización cuyo objetivo central es la igualdad de género en la escena musical uruguaya a través de la visibilización de las MyD músicas y la demanda de mecanismos de participación más equitativos. En el marco de sus acciones, la organización llevó a cabo el Primer Encuentro de Mujeres y Disidencias de la Música Uruguaya (PEMDMU, 2019) ${ }^{8}$ del cual surge buena parte de los datos que serán analizados a continuación.

\section{Consideraciones metodológicas}

El presente trabajo se valió de una caracterización cuantitativa descriptiva de la situación de mujeres y varones en el mercado laboral musical en base a los datos de Censos de Hogares, Población y Viviendas $\left(1985,1996^{9}\right)$, elaborados por Casacuberta y Roche (2001), puestos en diálogo con las Encuestas Continuas de Hogares (ECH, 2015-2018) en Uruguay. Una gran restricción constituye no contar con la variable identidad de género ${ }^{10}$. En el caso de los datos cuantitativos provenientes de fuentes oficiales, la unidad de análisis son las personas que hayan declarado ser músico/a ${ }^{11}$ o profesor/a de música como ocupación principal (aquella que reporta mayores ingresos). Ante esta selección se presenta como una limitación metodológica la posible subrepresentación de la población, ya que el trabajo está marcado fuertemente por el multiempleo y también por la dificultad de reconocer a la música como un trabajo. Sin embargo el análisis contribuye a caracterizar a quienes logran ser músicos/as como ocupación principal con representatividad estadística a nivel nacional.

También se analizaron los datos de la población encuestada mediante el formulario autoadministrado de inscripción al PEMDMU (2019) que contó con preguntas abiertas y cerradas. Por tanto, la muestra es de carácter no probabilístico, con lo cual no es posible

\footnotetext{
${ }^{8}$ En este marco se llevaron a cabo una serie de mesas de debate sobre las principales problemáticas de las mujeres y disidencias en la música y se aplicó un formulario de inscripción que permite contar con datos cuantitativos sobre las postulantes.

${ }^{9}$ Lamentablemente, los datos de Censo 2011 no permiten identificar a los/as músicas por el nivel de desagregación a solo 2 dígitos de tipo de ocupación. Por tal motivo, introducimos en el análisis la sumatoria de las encuestas continuas de hogares de 2015 a 2018 a fin de contar con un número de casos que permita hacer cruces de variables, manejando los porcentajes a partir de los promedios.

${ }^{10}$ Que ahora obliga la Ley Integral para Personas Trans, $\mathrm{N}^{\circ} 19.684$. Todos son anteriores al año de aprobación de la Ley (2018).

11 Incluye: músico, compositor de música, cantante de música, director de orquesta, director de banda musical, director de conjunto de carnaval, director de coro profesional, cantante de coro profesional, instrumentista musical, músico o cantor de cabaret, músico de orquesta, músico callejero, guitarrista, pianista, clarinetista, violinista, cellista, contrabajista, trompetista, trombonista, baterista, timbalista, intérprete musical y ejecutante de instrumento musical (Categorización de las ocupaciones basada en el Codificador Internacional Uniforme de Ocupaciones 08).
} 
realizar generalizaciones para toda la población de mujeres músicas y disidencias, aunque para la interpretación de los datos se ha intentado caracterizar, en relación a los datos representativos, al grupo que contestó el formulario mencionado. En el mismo se relevó información sociodemográfica de 226 personas, indagó sobre sus trayectorias educativas y laborales, sobre los géneros e instrumentos que interpretan, difusión y registro del trabajo y las percepciones de los obstáculos que identifican en su desarrollo profesional. El formulario preguntó identidad de género, y 91,7\% se identificó como "mujer cis"12, siendo "no binarias" (6,6\%) y "mujer Trans", "bisexual”, "género fluido" y "lesbianas" (1,7\%). El escaso número de casos $(8,3 \%)$ de personas con identidades de género disidentes, no permite profundizar en un análisis particular de este grupo. Las preguntas que abordan los obstáculos en el desarrollo de las trayectorias como músicas fueron de carácter abierto y fueron codificadas y agrupadas en categorías, acompañadas en su presentación por citas de las entrevistadas. De esta manera, hacemos explícitas las limitaciones de los datos, esperando que los avances normativos impacten en el enriquecimiento del conocimiento sobre las desigualdades de género más allá de una visión genérica desprendida de la dicotomía varón/mujer.

\section{Música, participación y desigualdades de género}

El análisis de los Censos de 1985 y 1996 y los datos de las ECH 2015-2018 permiten conocer que dentro de los distintos trabajos artísticos, el sector de la música es el más popular $^{13}$, aunque su peso en el sector ha decrecido. Además, la música es el sector más masculinizado dentro de lxs trabajos artísticos.

Gráfico 1. Masculinización del trabajo artístico. ECH, 2015-2018, Uruguay

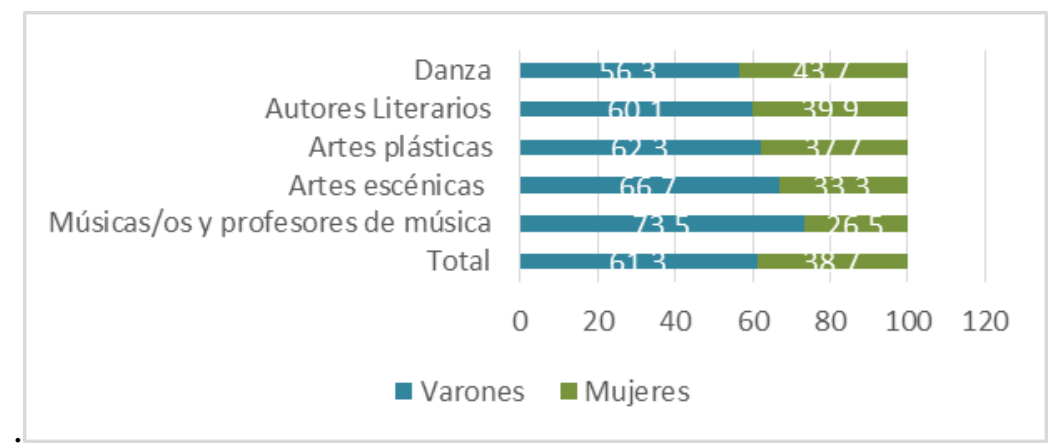

$12 \mathrm{Su}$ identidad de género coincide con su fenotipo sexual.

${ }^{13}$ El codificador de ocupaciones permite generar categorías más o menos desagregadas en relación a las tareas. Para lograr comparabilidad con los estudios de Casacuberta y Roche (2001) consideramos como grandes categorías de artistas a: autores literarios; pintores, escultores y grabadores; músicos (directores de orquesta, coros, compositores, instrumentistas, profesores de música); bailarines, directores de danza y coreógrafos y artes escénicas (directores de escena, actores, comediantes, artistas de circo, TV y radio, teatro). 
Fuente: elaboración propia de los microdatos de las Encuestas Continuas de Hogares 2015-2018, Instituto Nacional de Estadística, Uruguay.

Si bien se observa una leve tendencia al aumento de la participación de las mujeres cuya ocupación principal es ser artistas en el período 1985 a 2018, esto no sucede para el caso de la música. En el período mencionado, las directoras de orquesta, coros, compositoras, instrumentistas y profesoras de música disminuyeron levemente; pasaron de 28,3\% en 1985 a 26,5\% en 2015-2018. Las pintoras, escultoras y grabadoras, se han mantenido relativamente estables en el período considerado (38,8\% en 1985 a 37,7\% en 2015-2018). Aumentaron las mujeres que trabajan como autoras literarias (de 32,8\% a 39,9\%) y las directoras de escena, actrices, comediantes, artistas de circo, TV y radio teatro $(31,4 \%$ a $33,3 \%)$.

Respecto a la segregación vertical, se observa que las mujeres participan mucho menos y en puestos de menor reconocimiento. La música como parte del ámbito público ha sido habitada por los varones de manera concomitante con la división sexual del trabajo tradicional (AGUIRRE, 2009; BATTHYÁNY, 2015; MURILLO, 1998). Aquí la participación es una temática central. El empoderamiento ${ }^{14}$ dentro de los espacios masculinos es un reto para las mujeres y disidencias, debido al blindaje que tiene la música como espacio masculinizado de desempeño (LIMA, 2013) y a las incompatibilidades de la organización del trabajo musical con el trabajo para la sostenibilidad de la vida. Participar (estar ahí, decidir, negociar, construir) o no participar, es un primer paso para debatir luego "la calidad" de las modalidades de participación. Los antecedentes señalan fuertes restricciones para la participación de las mujeres y disidencias en la música. El colectivo Ruidosa analizó para el caso latinoamericano la participación en 2017 de mujeres en festivales de música en Colombia, Chile, México, Argentina y Estados Unidos. En todos los países la participación de mujeres sin varones en el escenario fue de $10,1 \%$ en 2017 , siendo $11,3 \%$ el total de las bandas mixtas en los espectáculos. Los datos de Women in Music plantean que la participación de las mujeres en la música es del orden del $30 \%{ }^{15}$. Del total de los productores reconocidos, $6 \%$ son mujeres. En las sociedades de gestión colectiva las mujeres representan un $20 \%-\mathrm{o}$ menos- del total de los compositores registrados. El 22\% de las personas que trabajaron en la elaboración de las 600 canciones más populares entre 2012 y 2017 fueron mujeres y representan solo el $12 \%$ del total de compositores de las 600 canciones más populares en dicho período.

\footnotetext{
${ }^{14}$ Proceso por el cual los individuos (en este caso las mujeres) conquistan paulatinamente la capacidad de participación y acción autónoma.

${ }^{15}$ https://www.womeninmusic.org/
} 
Cuadro 1. Resumen de las dimensiones segregación vertical, selectiva y horizontal como expresiones de la división sexual del trabajo.

\begin{tabular}{|c|c|}
\hline $\begin{array}{l}\text { Segregación vertical: las demandas de } \\
\text { participación en la música }\end{array}$ & $\begin{array}{l}\text { Masculinización de la actividad (84\% varones y } 16 \% \text { mujeres en su muestra) } \\
\text { (Casacuberta y Roche, 2001). } \\
\text { El } 80 \% \text { de los músicos son varones (González, 2009). } \\
\text { 2015-2018). } \\
\text { 73,5\% de las personas cuya ocupación principal es la música son varones (ECH } \\
\text { El 27\% del total de las problemáticas enunciadas por las encuestadas del PEMDMU } \\
\text { refieren a la participación. } \\
\text { "No suele haber espacios para mujeres y disidencias", "Pocos espacios en los } \\
\text { festivales públicos", "La dificultad de acceso a espacios", "Los espacios generados } \\
\text { para nuestra música son pocos". } \\
\text { Falta de capital social y comunicación (desconfianza por parte de los contratantes, } \\
\text { desvalorización del trabajo). } \\
\text { Ausencia o escasas invitaciones para tocar. Dificultades en la difusión y manejo de } \\
\text { las redes que las pueden colocar en lugares más visibles (desconocimiento de } \\
\text { gestores que arman las grillas, amplificación de la difusión de su trabajo y acceso a } \\
\text { medios de comunicación). } \\
\text { "Abundan las propuestas para los hombres, en los festivales siempre son varones y } \\
\text { con suerte alguna mujer corista", "En Uruguay las artes siempre están en un } \\
\text { segundo plano, y al ser mujer, menos oportunidades se tienen, "No hay muchos } \\
\text { lugares para hacerse conocer", "Se abre poco espacio a las mujeres, y hay muchas } \\
\text { músicas en Uruguay". }\end{array}$ \\
\hline Segregación selectiva & $\begin{array}{l}\text { Perfil más joven en relación al resto de las personas ocupadas, blancas, de niveles } \\
\text { educativos y económicos más altos en relación al total de la población }\end{array}$ \\
\hline $\begin{array}{c}\text { Segregación horizontal: el desplazamiento } \\
\text { de roles de género tradicionales al ámbito } \\
\text { musical }\end{array}$ & $\begin{array}{l}\text { 80\% de los músicos son varones, 80\% de los profesores de música son mujeres } \\
\text { (GONZÁLEZ, 2009, p.6-9). } \\
\text { Las mujeres participan más en la música cuando enseñan (39,5\%), en relación a las } \\
\text { personas que tocan o viven como instrumentistas (16,9\%). } \\
\text { "En el área de la docencia los centros educativos priorizan las figuras masculinas } \\
\text { en el área de la educación musical”. } \\
\text { La menor participación de las mujeres en la categoría "técnicos de sonido y } \\
\text { profesiones similares a DJs" (12,7\%) (IM-RDM, 2018, p.10). } \\
\text { División de roles en las murgas } 16 .\end{array}$ \\
\hline
\end{tabular}

Nota: en cursiva, citas de las entrevistadas por el PEMDMU.

Las mujeres que consiguen que la música sea su ocupación principal tienen un perfil específico que hace pensar en una segregación selectiva. El perfil de quienes tienen como ocupación principal la música, como de las personas encuestadas para el PEMDMU, es coincidente con las características señaladas por los antecedentes para lxs trabajadores del ámbito de la cultura. Por tanto, es importante considerar los posicionamientos

16 El porcentaje de mujeres que integran murgas (cantando o tocando la batería) en 2017 fue de 3,6\% ascendiendo a 5,9\% en 2018. No hubo en ninguno de los dos años directoras escénicas mujeres en el Concurso Oficial del Carnaval. En la tarea de letristas, solo 1,5\% del total en 2017 eran mujeres y 3,8\% en 2018. Ninguna mujer participó realizando arreglos musicales en 2017 o 2018 en las murgas en el Concurso Oficial. Sin embargo, en Vestuario en 2017, el 72,0\% eran mujeres disminuyendo a 46,5\% en 2018 y en maquillaje, 64,0\% de las maquilladoras artísticas eran mujeres en 2017 y 52,6\% en 2018. Fuente: elaboración propia en base a datos sistematizados de los componentes de las murgas en las fichas técnicas del sitio http://carnavaldeluruguay.com/ 
socioestructurales de lxs encuestados a la hora de comprender a quiénes representan los discursos y las demandas expresadas por el colectivo analizado: mujeres universitarias, artistas, mayoritariamente blancas y montevideanas de niveles educativos altos y económicos medios-altos y altos.

Por otra parte, se observa la presencia de segregación horizontal basada en género. La misma remite a la observación sobre si la distribución de los roles y funciones de las personas en una actividad imitan un patrón (por ejemplo los roles de género tradicionales), para luego indagar acerca de la valorización simbólica y económica que tienen las diferentes actividades (KERGOAT, 2009). Una idea básica en la segregación horizontal de género, es que son reservados para las mujeres aquellos lugares que se asocian con las responsabilidades domésticas, de cuidados o con la sexualización de sus cuerpos. En el mercado laboral, en general, se encuentra una fuerte feminización de ámbitos de trabajo de cuidados como el ámbito educativo, la psicología, el trabajo social, la salud, no así en los ámbitos vinculados a la industria y el comercio, la diplomacia, entre otros (INMUJERES, 2017). Sumado a lo sistematizado en el cuadro anterior, se puede observar segregación horizontal en el caso de las músicas respecto a los instrumentos interpretados, que también habla acerca de los roles en los grupos musicales. La amplia mayoría de las personas que contestaron el formulario PEMDMU tocan 1 o 2 instrumentos (66\%), siendo $29,6 \%$ que mencionan entre 3 y 6 instrumentos y un porcentaje menor que tocan 6 instrumentos y más $(4,4 \%)$. Los instrumentos que más mencionan las mujeres son voz y guitarra, obedeciendo a la imagen de front-woman. Sin embargo, fue mencionada una amplia gama de instrumentos que tocan las mujeres y disidencias, lo cual contribuye a romper estereotipos de género en la música y generar nuevos modelos de referencia para las mujeres más jóvenes.

Tabla 1. Tipo de instrumentos que tocan mencionados por las participantes del PEMDMU, 2019.

(Respuesta múltiple).

\begin{tabular}{|c|c|}
\hline & Porcentaje \\
\hline Total cordófonos & 28,1 \\
\hline Total electrónicos & 2,9 \\
\hline Total percusión & 36,9 \\
\hline Total ventos & 31,9 \\
\hline Ninguno & 0,2 \\
\hline Total & 100 \\
\hline
\end{tabular}

Fuente: Elaboración propia en base a los microdatos del Formulario Autoadministrado del Primer Encuentro de Mujeres y Disidencias de la Música en Uruguay. 


\section{Características de la música como trabajo: precariedad laboral y desigualdades de género}

Tal como fue señalado en los antecedentes, el trabajo artístico presenta grados de precarización que se expresan en varias dimensiones: económica, temporal, organizacional y social (ESOPE, 2005). A continuación se resumen los datos cuantitativos y cualitativos que refieren a las distintas dimensiones, con perspectiva de género.

Cuadro 2. Datos cuantitativos y cualitativos para las dimensiones de precariedad laboral propuestas por ESPOE (2005).

\begin{tabular}{|c|c|}
\hline $\begin{array}{l}\text { TEMPORAL: refiere al } \\
\text { grado de certeza en la } \\
\text { continuidad del empleo }\end{array}$ & $\begin{array}{l}\text { Buena parte }(45,1 \%) \text { de las personas que tienen como ocupación principal ser músicas/os en la ECH } 2015-2018 \text {, son } \\
\text { cuentapropistas con local o inversión. Hay una mayor presencia de asalariados públicos entre quienes declaran a la } \\
\text { música como ocupación principal. } 32,8 \% \text { son asalariados públicos y } 16,9 \% \text { privados. Levemente mayor } \\
\text { participación como miembros de cooperativas de producción o trabajo }(1,6 \%) \text { en relación al total de activos }(0,2 \%)\end{array}$ \\
\hline $\begin{array}{r}\text { ORG } \\
\text { con } \\
\text { colectiv }\end{array}$ & $\begin{array}{l}\text { equidad de género en el trabajo desde } 2002 \text { hasta el pres } \\
\text { capacidad de incidir en las políticas públicas y la distrib } \\
\text { socio-p }\end{array}$ \\
\hline & $\begin{array}{l}\text { De las personas encuestadas en el PEMDMU solo } 6,6 \% \text { son asalariadas. Un } 31 \% \text { tiene contrato cachet; } 11,1 \% \\
\text { cubierto artístico; } 4,4 \% \text { trabaja "a voluntad", } 8 \% \text { porcentaje por entradas en la puerta: } 0,4 \% \text { jubilada y } 25,7 \% \text { trabaja } \\
\text { de manera no remunerada. Un } 12,4 \% \text { no respondió "No poder tener un ingreso ni aportar por mi trabajo. La } \\
\text { inestabilidad del mismo", "Gran dificultad para cobrar por tocar", "Pocas veces remunerado", "Pocos lugares } \\
\text { donde presentarse y que paguen", "Que te ofrezcan un pago digno". "No soy remunerada acorde a mi trabajo" } \\
\text { Ausencia de progresión salarial y brecha salarial de género: Se estimó una brecha de } 9,2 \% \text { entre varones y mujeres } \\
\text { cuya ocupación principal es ser músicos/as. La misma es menor }(9,2 \%) \text { al total de la brecha de ingresos por } \\
\text { ocupación principal del total de los activos }(27,8 \%) \text {. }\end{array}$ \\
\hline $\begin{array}{l}\text { SOCIAL: protección legal } \\
\text { contra despidos injustos, } \\
\text { discriminación en el trabajo, } \\
\text { protección social } \\
\text { (beneficios salud, seguridad } \\
\quad \text { social, desempleo, } \\
\text { accidentes laborales) }\end{array}$ & $\begin{array}{c}\text { La mayor proporción de trabajadores autónomos afecta la capacidad de gozar de algunos derechos que se canalizan a } \\
\text { través del trabajo asalariado. La ausencia de cultura de contrato (Varela, 2018), trabajo a la gorra, cachet, } \\
\text { contrataciones puntuales imposibilitan protección social. Altos niveles de informalidad: Del total de las personas } \\
\text { cuya ocupación principal es la música, } 49,7 \% \text { no realiza aportes a cajas jubilatorias, a diferencia del total de los } \\
\text { activos, en los que solo un } 22,9 \% \text { no declara aportes jubilatorios. } \\
\text { La calidad del empleo es la principal problemática mencionada por las encuestadas del PEMDMU (concentra el } \\
36 \% \text { de las problemáticas enunciadas), seguida de "Discriminación basada en género" (31\%) y la ya mencionada } \\
\text { "falta de oportunidades y visibilidad" (27\%). }\end{array}$ \\
\hline
\end{tabular}

Nota: en cursiva, citas de las entrevistadas por el PEMDMU.

En cuanto a la dimensión temporal, el trabajo por cuenta propia marca que el grado de incerteza de continuidad en el empleo sea alto, ya que la protección social es más débil en relación a los asalariados. Esto representa serias limitaciones para el caso de las mujeres en las etapas reproductivas, puesto que no siempre están previstas licencias por maternidad, ni por cuidados cuando un familiar enferma o necesita apoyo. Las características del empleo informal en el caso de las mujeres músicas, no sólo afecta su autonomía económica, la posibilidad de desarrollo de sus carreras, sino que además afecta su futuro, la magnitud de los montos jubilatorios (AGUIRRE y SCAVINO, 2018).

A nivel organizacional, si bien las mujeres participan en menor proporción en la música como ocupación principal, tal como se ha mostrado en los antecedentes, mujeres y 
disidencias llevan adelante organizaciones sociales que buscan incidir en el debate público sobre derechos laborales y equidad de género.

En cuanto a la dimensión económica, el no pago o pago insuficiente es una de las grandes temáticas que preocupan a las entrevistadas, de las cuales una de cada cuatro no cobra por su trabajo. Además, se mencionan como fuentes de cobro el cachet, o el arreglo de ganancia según las entradas vendidas, o según lo propuesto por el empleador. También hay ausencia de progresión salarial en la medida en que la mayoría no son asalariados y no hay acuerdos de fijación de precios previa. La percepción de la inseguridad económica, el reclamo de salarios y de precios fijos para el cachet, son en esta organización contrarios a la hipótesis de Bourdieu (1997), acerca del desinterés económico y la ponderación de la vocación y el placer que reporta el trabajo artístico como moneda de cambio en la circulación de capitales económicos y simbólicos. La ausencia de una cultura del contrato o de la existencia de pautas comunes y acordadas para la retribución y formas de contratación es una problemática central en la configuración y profesionalización del trabajo en la música (VARELA, 2018). Además, se calculó una brecha salarial de género que indica que las mujeres ganan 9,2\% menos que los varones, lo cual puede implicar mayores niveles de pagos insuficientes. Las entrevistadas lo asocian a lo que perciben como una sobre-exigencia: "En general cuando se llega a un lugar históricamente identificados por hombres hay que «demostrar» estar a la «altura»" y sienten "La necesidad de justificar el doble o triple mi trabajo que un varón".

Por otra parte, los altos niveles de informalidad indican la ausencia de la protección social a través del empleo, lo cual ante etapas reproductivas deja sin protección laboral a las mujeres. Tampoco permite que las MyD accedan a los mecanismos de corresponsabilidad en los cuidados canalizados a través de empleos formales y asalariados, principalmente.

\section{Principales problemáticas en la música como profesión en las encuestadas del PEMDMU}

La gráfica 2 presenta una porcentualización de las respuestas de las preguntas abiertas que fueron codificadas en el análisis. La principal dificultad refiere a la calidad del empleo (36\%), seguida de la discriminación basada en género (31\%), la falta de oportunidades y visibilidad (27\%), falta de recursos económicos (5\%) y dificultad en el acceso a espacios de formación (1\%). 
En el apartado anterior fueron integradas algunas de las citas acerca de la calidad del empleo (dimensión económica y social de la precariedad laboral) y la falta de oportunidades y visibilidad (vinculada con la segregación vertical).

Gráfica 2. Problemáticas en el trabajo musical, cantidad de respuestas por categoría en porcentaje.

PEMDMU, 2019.

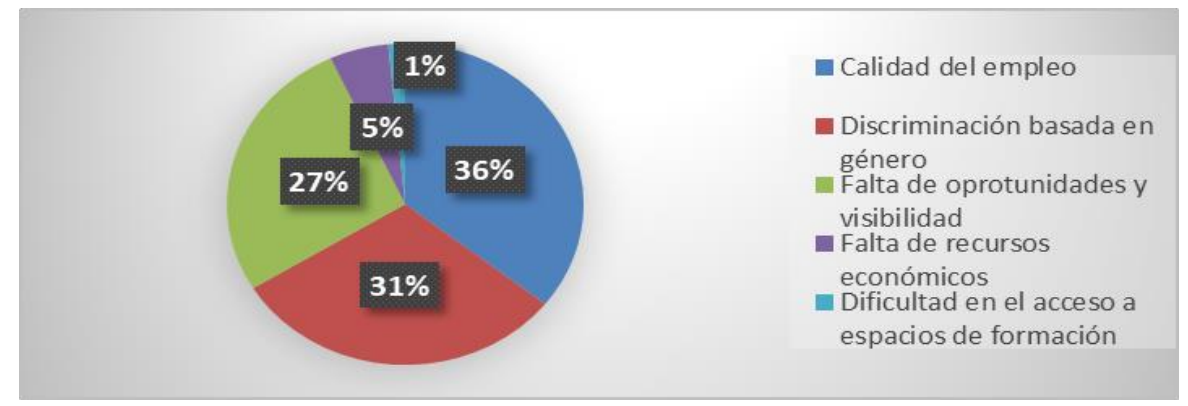

Fuente: Elaboración propia en base a los microdatos del Formulario Autoadministrado del Primer Encuentro de Mujeres y Disidencias de la Música en Uruguay. Nota: el total de citas codificadas fue 261. Para el procesamiento de las categorías se utilizó el software Atlas Ti.

En cuanto a la calidad del empleo, se señala al multiempleo como limitación para el desarrollo como profesionales en la música: "El cansancio que genera hacer todo (armar afiches, eventos, hablar con diferentes personas, etc."; "Tener que encargarme de trabajos no musicales para generar público, gestión, etc”. También se menciona la escasez de recursos para contar con los soportes técnicos necesarios para tocar, lo cual es una problemática que forma parte de la calidad del trabajo "Conseguir espacios de ensayo, hacerse de los instrumentos para la investigación, amplificación en los espacios de presentaciones", "Escasez de recursos al momento de grabar o producir".

Como adelantamos, es importante mencionar que las mujeres muestran bajos niveles de registros de sus obras, viéndose afectada la difusión y el registro de su trabajo (GUIRAO, 2019). En 2008, de un total de 142 discos editados, 114 fueron de solistas o de agrupaciones musicales. Entre estos últimos, solamente un $8 \%$ eran mujeres solistas o grupos de mujeres, $15 \%$ eran grupos mixtos y el $78 \%$ restante, solistas o agrupaciones exclusivamente integradas por varones (GONZÁLEZ, 2009, p.6-9). De las personas que contestaron el formulario del PEMDMU, 45,1\% no han grabado o editado singles largos o cortos y sí lo hicieron el 39,1\%, siendo la mayoría grabaciones independientes. Un 15,9\% del total declara que está en proceso de edición por primera vez. Por otro lado, más de la mitad de las participantes $(55,3 \%)$ tampoco tiene participación en fonogramas como invitadas y el 30,1\% que lo hizo fue 
también en un formato independiente, siendo las menos quienes grabaron como invitadas en sellos discográficos.

Tabla 3. Grabación, producción y edición de discos o singles y participación como invitadas en proyectos, PEMDMU, 2019.

\begin{tabular}{|c|c|c|c|}
\hline & & Cantidad & Porcentaje \\
\hline \multirow{5}{*}{ Edición de discos o singles } & Estoy en proceso de editar por primera vez & 36 & 15,9 \\
\hline & No & 102 & 45,1 \\
\hline & $\mathrm{Si}$, con un sello discográfico & 29 & 12,8 \\
\hline & $\mathrm{Si}$, de manera independiente & 59 & 26,1 \\
\hline & Total & 226 & 100 \\
\hline & & Cantidad & Porcentaje \\
\hline \multirow{4}{*}{ Grabaron como invitadas } & No & 125 & 55,3 \\
\hline & $\mathrm{Si}$, de manera independiente & 68 & 30,1 \\
\hline & Sí, en un sello discográfico & 33 & 14,6 \\
\hline & Total & 226 & 100,0 \\
\hline
\end{tabular}

Fuente: Elaboración propia en base a los microdatos del Formulario Autoadministrado del Primer Encuentro de Mujeres y Disidencias de la Música en Uruguay.

Finalmente, la segunda dimensión más mencionada (31\%) por las encuestadas del PEMDMU es la discriminación basada en género. Dentro de ésta podemos ubicar a la discriminación basada en género como maltrato y prejuicios asociados a la identidad de género y mansplaining ${ }^{17}$ (18\%), la ausencia de corresponsabilidad y pobreza de tiempo (11\%) y el acoso sexual (2\%). Las mismas también aparecieron en los discursos recogidos en varios antecedentes (IM, 2019; IM-RDM, 2018; BONINO, 2018; CASTRO, 2018; ESPASANDÍN, 2012).

Cuadro 3. Porcentaje de citas y citas en sub-dimensiones dentro de Discriminación de Género. Atlas Ti. PEMDMU, 2019.

\begin{tabular}{|c|c|}
\hline $\begin{array}{l}\text { Desvalorización del } \\
\text { trabajo, maltrato y } \\
\text { mansplaining }(17,6 \%)\end{array}$ & $\begin{array}{l}\text { "la eterna duda de no saber si se te respeta por la música o la apariencia”, "es difícil que te } \\
\text { tomen en cuenta, soy más criticada", "cuando tenés una idea nueva para una canción o una } \\
\text { melodía muchas veces se te mira mal o no se te toma en cuenta", "soy la mala onda si quiero } \\
\text { aprovechar las horas de ensayo", "sonidistas con mal trato y manejando un mal tono o } \\
\text { tratándote como si no supieras nada", "invisibilidad, mala paga y mansplaining”, "machismo } \\
\text { que evalúa si somos o no capaces, que deslegitima nuestra música, que les parece que lo hacemos } \\
\text { como pasatiempo y no es así, que no se cansan de explicarnos cómo y qué hacer". }\end{array}$ \\
\hline $\begin{array}{c}\text { Ausencia de } \\
\text { corresponsabilidad y } \\
\text { pobreza de tiempo } \\
(11,5 \%)\end{array}$ & $\begin{array}{l}\text { "actualmente la maternidad"; "actualmente lo central es la maternidad sola, asociada al autismo } \\
\text { de mi hijo, que hace que no pueda llevarlo a lugares ni dejarlo al cuidado de alguien así nomás } \\
\text { (por ejemplo para este evento, más allá de que aplaudo la iniciativa del espacio recreativo, que } \\
\text { de seguro le va a hacer posible la participación a muchas madres)"; "en este momento la crianza } \\
\text { de mis hijos", "dejar a mi hijo a cuidado de otros durante los shows nocturnos, o dias de } \\
\text { grabación", "tiempo, compatibilizar responsabilidad maternal, posibilidad de desarrollo } \\
\text { profesional frente al escaso tiempo de dedicación a ensayo y trabajo en hogar", "los espacios, el } \\
\text { tiempo, recursos económicos para concretar proyectos, gestión, difusión, tiempos disponibles con } \\
\text { la maternidad (hijo de } 21 \text { meses)"” }\end{array}$ \\
\hline
\end{tabular}

\footnotetext{
${ }^{17}$ Mansplaining: el acto de explicarle algo a alguien de una manera que sugiere que es tonta/o; se usa especialmente cuando un hombre le explica algo a una mujer que ella ya comprende o vive mientras el hombre no.

Fuente: https://dictionary.cambridge.org/es-LA/dictionary/english/mansplaining
} 


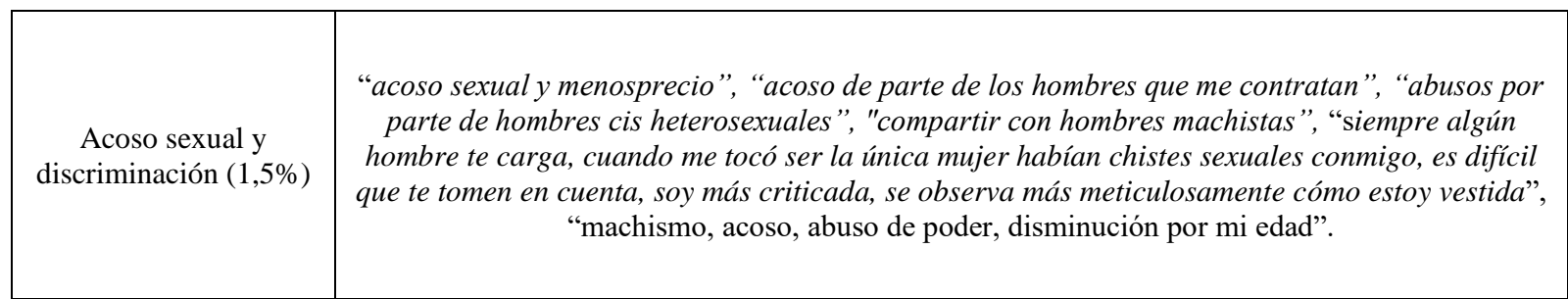

Fuente: Elaboración propia en base a los microdatos del Formulario Autoadministrado del Primer Encuentro de Mujeres y Disidencias de la Música en Uruguay. Nota: el total de citas codificadas fue 261.

Una de las grandes limitaciones para la incorporación de las mujeres al mercado laboral son las complicaciones de tiempo que tienen por asumir en mayor medida que sus pares varones las responsabilidades por el trabajo doméstico y de cuidados. Este aspecto aparece referenciado en el $11 \%$ de las citas que refieren a las problemáticas que las MyD experimentan en su desarrollo professional en la música. Del total de las personas encuestadas en el PEMDMU, el 30,5\% convive con hijos y 1,4\% con personas en situación de dependencia. Para ellas, el tiempo para dedicarse a la música no solo entra en competencia con el tiempo que se dedica a otros empleos más rentables, sino también a los cuidados, la maternidad y las tareas domésticas. El hecho de que la maternidad sea concebida como un problema en relación al desarrollo de otros proyectos centrales en la vida de las mujeres es un indicador de cómo opera actualmente un sistema de división sexual del trabajo que afecta principalmente a las mujeres. La maternidad no es un problema en sí, lo que resulta problemático es la organización de la actividad música, su configuración como empleo y profesión, así como la organización social de los cuidados, basadas en el trabajo no remunerado de las mujeres de las familias (OIT, 2018; ONU MUJERES, 2017; BATTYÁNY, 2015). La organización de la música como actividad, los horarios de ensayo y espectáculos, fueron construidas en torno a la idea de un sujeto libre de responsabilidades de cuidados y domésticas. En su búsqueda por desarrollarse en la música, las mujeres expresan como un aspecto problemático la incompatibilidad de tiempos de cuidados y trabajo ${ }^{18}$.

Las problemáticas en torno a la corresponsabilidad en el caso de las mujeres pueden vincularse a los arreglos de convivencia. Los varones músicos viven solos en mayor proporción que las mujeres (16,9\% vs. 9,8\%) y que el total de varones activos en el mercado laboral $(8,4 \%)$. Por el contrario, las mujeres que tienen como ocupación principal ser músicas conviven con parejas e hijos (de ambos o solo de uno) en mayor proporción que los varones (40,3\% vs 36,4\%). Esto es importante para comprender la mayor participación de los varones

\footnotetext{
${ }^{18}$ A su vez, la escasa cobertura universal para niños/as menores de 4 años y la necesidad de mayores escuelas a tiempo completo son un aspecto que impacta en las trayectorias laborales de las mujeres, a diferencia de los varones (BATTHYÁNY, GENTA y SCAVINO, 2018).
} 
en la música, ya que dicho empleo tiene características dinámicas, horarios nocturnos, requiere la capacidad de viajar, o de ensayar a altas horas de la noche, lo cual se vincula con la "libertad" en el manejo de los tiempos.

\section{Reflexiones finales}

La histórica masculinización del sector musical está indicada a partir de diferentes mediciones que muestran que las mujeres nunca llegan a ser ni un tercio del total de las personas que se desempeñan en la música. Se trata del sector que reúne a más personas del total de los sectores artísticos, y a su vez, es el más masculinizado. Los datos indican que desde 2015 a 2018, en promedio las mujeres representaron el 26,5\% del total de las personas que consiguen que la música sea su ocupación principal, y, mientras que en otras ramas del arte ellas tienden a aumentar su participación, en el caso de la música esta tendencia no se evidencia.

Existe, asimismo, una segregación en el acceso a la música como actividad, dentro de la cual se detecta una segregación selectiva: logran trabajar en la música mujeres y disidencias que se caracterizan por tener niveles educativos medios-altos, pertenecer a hogares de niveles económicos medios-altos y altos, ser mayoritariamente blancas, de Montevideo y más jóvenes en relación al total de las mujeres activas. La segregación de las mujeres y disidencias con menos recursos económicos es señalada como una problemática en los intentos por desarrollar una carrera en la música.

A su vez, existen patrones de género en los ámbitos en los que se desempeñan las mujeres y los varones que son diferenciados. La segregación horizontal en la música se expresa, por ejemplo, en que las mujeres logran trabajar enseñando música $(39,5 \%)$ en mayor proporción que las que logran trabajar como intérpretes, compositoras, instrumentistas (16,9\%). También se observa en el hecho de que las mujeres y disidencias tienen un menor desarrollo en lugares técnicos de la música (sonidistas, productores, managers, entre otros) que están masculinizados, siendo un lugar difícilmente habitable por las músicas. Al mismo tiempo, los aspectos más vinculados a la ornamentación del cuerpo (vestimenta y maquillaje), como a los cuidados (enseñar) están feminizados.

En cuanto a las principales problemáticas identificadas por las encuestadas PEMDMU, se menciona en primer lugar la calidad del empleo, seguida de la discriminación basada en género que se desagrega en tres dimensiones: maltrato y mansplaining, ausencia de corresponsabilidad y escasez de tiempo y acoso sexual (2\%). Como ha sido mencionado, la 
falta de recursos económicos y la dificultad en el acceso a espacios de formación también son problemáticas emergentes.

Los discursos exponen que el ámbito profesional de la música es hostil con las mujeres y disidencias, perpetuando formas tradicionales de discriminación de género como el menosprecio, la ausencia de reconocimiento del conocimiento y trabajo de las mujeres y la sobre-explicación. Los tiempos provenientes de las dobles o triples cargas de trabajo en el caso de las mujeres y disidencias en las músicas, son un impedimento para su desarrollo en el ámbito. La configuración de la música como una actividad de varones y para varones hace que la nocturnidad del trabajo (ensayos y espectáculos), las giras, y las dinámicas en torno a la actividad se presenten como incompatibles con responsabilidades domésticas y de cuidados. Al ser las mujeres quienes socialmente siguen encargándose de estas tareas, la configuración de la actividad musical y la ausencia de mecanismos de corresponsabilidad afectan la inclusión, participación y desarrollo de las mujeres y disidencias en la música.

El estudio muestra que la calidad del empleo presenta altos niveles de informalidad y precarización. La mayoría de las personas que trabajan en la música lo hacen por cuenta propia, sin una institucionalidad o apoyo en la protección de riesgos laborales. El trabajo en el sector musical presenta problemas en cuanto a su formalidad y la potencialidad de acceder a los derechos canalizados a través del mismo: 1 de cada 2 personas músicas no aportan a ninguna caja de jubilaciones, según los datos de las ECHs. Si bien varones, mujeres y disidencias enfrentan estas mismas problemáticas en el sector, se ha constatado una brecha salarial entre músicos varones y mujeres, que indica que las mujeres ganan 9,2\% menos que los varones. A su vez, 1 de cada 4 personas encuestadas por el PEMDMU no percibe remuneración a cambio de su trabajo.

Por otra parte, en cuanto al registro y difusión del trabajo, los estudios antecedentes muestran que las mujeres editan y graban menos discos que los varones (GONZÁLEZ, 2009; CASACUBERTA y ROCHE, 2001) y en el perfil de las encuestadas del PEMDMU, a pesar de que más del $70 \%$ cuenta con 6 años o más de experiencia en la música, casi la mitad $(45,1 \%)$ no han registrado su trabajo y un $15,9 \%$ estaba en proceso de hacerlo por primera vez. El multiempleo y la falta de infraestructura para tocar y ensayar fueron mencionadas como problemáticas asociadas a la calidad del empleo. Como obstáculos para la profesionalización en la música, se señaló la privatización de la formación y la falta de recursos económicos.

En el análisis de los datos, se hizo evidente que es necesario un mayor desarrollo de estudios que sistematicen el conocimiento de las personas que trabajan en el ámbito cultural, 
tanto cuantitativos como cualitativos. La inexistencia de encuestas oficiales que permitan conocer con mayores niveles de profundidad las desigualdades de género en la música es una gran limitante para la detección de problemáticas sociales. A su vez, es necesario avanzar sobre la idea de trayectorias, en el entendido de que observar a las mujeres que ya están ocupadas en la música supone dejar fuera del análisis a aquellas que quisieron desempeñarse como tales y no pudieron lograrlo.

Por último, resulta ineludible mencionar que el contexto actual de la pandemia desatada a nivel mundial por el COVID-19, desnuda las condiciones de precarización y flexibilización laboral a la que están expuestos lxs trabajadores de la cultura desde tiempo atrás. Son numerosas las iniciativas internacionales y nacionales que se están llevando a cabo desde diferentes organismos públicos, asociaciones civiles, organizaciones de artistas en todo el mundo para plantear medidas paliativas a la preocupante situación que atraviesan. En ese sentido, resultaría esperable que en la visibilización de lo que en contextos "normales" está altamente invisibilizado, las condiciones del trabajo artístico entren en agenda pública y se posibilite el desarrollo de investigación, así como implementación de políticas regulatorias hacia estos trabajos, atendiendo sus especificidades. En paralelo, también se constata el avance de los movimientos sociales que buscan la equidad de género en el ámbito de la cultura, particularmente de la música, la generación de información y las luchas por el desarrollo de políticas sociales. Apostamos a que estos avances abran camino hacia la modificación de los estereotipos y roles de género que limitan el pleno desarrollo de la ciudadanía social de mujeres y disidencias.

\section{Bibliografía}

AGUIRRE, R. "Uso del tiempo y desigualdades de género en el trabajo no remunerado", en AGUIRRE, Rosario (ed.) Las bases invisibles del bienestar social. El trabajo no remunerado en Uruguay, Montevideo, Doble Clic Editoras, 2009, pp. 23-81.

AGUIRRE, R., y SCAVINO, S. Vejeces de las mujeres. Desafíos para la justicia social y la equidad de género en Uruguay, Montevideo, Doble Clic Editoras, 2018, pp. 171.

BATTHYÁNY, K., GENTA, N., y PERROTTA, V. (2015). "Uso del tiempo y desigualdades de género en el trabajo no remuenrado", en BATTHYÁNY, Karina, Los tiempos del bienestar social. Género, trabajo no remunerado y cudiados en Uruguay, Montevideo, Doble Clic, pp. 87-132.

y SCAVINO, S. "División sexual del trabajo en Uruguay en 2007 y 2013. Tendencias en los cambios y en las permanencias de las desigualdades de género", en Revista Austral de Ciencias Sociales 32, 1, 2017, pp.121-142. 
GENTA, N., y SCAVINO, S. "Cambios y permanencias en las estrategias de cuidado infantil en el curso de vida: un análisis de género", en $O$ social en questao, 21 (43), 2019, pp. 25-120.

, Los tiempos del cuidado en Uruguay. Los tiempos del bienestar social. Género, trabajo no remunerado y cuidados en Uruguay, Montevideo, Doble Clic Editoras, 2015.

GENTA, N., y PERROTTA, V. La población uruguaya y el cuidado. Análisis de representaciones sociales y propuestas para un Sistema de Cuidados en Uruguay. Montevideo, Ministerio de Desarrollo Social, 2013.

BECK, A. Cultural Work. Understanding the cultural industries. Londres, Routledge, 2003.

BIANCHI, S., MILKIE, M., SAYER, L., y ROBINSON, J. "Is anyone doing the housework? Trends in the gender division of household labor", en Social Forces 79,1, 2000, pp. 191-228.

BONINO, S. "Género y música en Uruguay”, en VARELA, M. (coord.) Apuntes 1.0. Músic@s yColectivos, Montevideo, Fans de la Música, 2018, pp. 17-22.

BOURDIEU, P. Razones prácticas. Sobre la teoría de la Acción. Barcelona, Anagrama, 1997.

BRAIDOTTI, R. Patterns of Dissonance, United Kingdom, Polity Press, 1991.

BRODSKY, J., NEGRÓN, B., y POSSEL, A. El escenario del trabajador cultural en Chile. Proyecto Trama / Observatorio Políticas Culturales, 2014.

BUTLER, J. El género en disputa. El feminismo y la subversión de la identidad, Buenos Aires, Paidós, 2001.

CARRASCO, C. BORDERÍAS, T. y TORNS, T., "Introducción" en CARRASCO, C. BORDERÍAS, T. y TORNS, T. (eds.) El trabajo de cuidados. Historia, teórica y políticas. Madrid, Los libros de la catarata, 2011, pp. 11-91.

BORDERÍAS, T., y TORNS, T. El trabajo de cuidados. Historia, teórica y políticas, Madrid, Los libros de la catarata, 2011, pp. 11-91.

CASACUBERTA, C., y ROCHE, H. El mercado laboral y la carrera de los músicos en Uruguay. Montevideo, SUDEI-UDELAR, 2001.

CASTRO, S. "Mujeres en la música", en VARELA, M. (coord.) Apuntes 1.0. Músic@s y Colectivos, Montevideo, Fans de la Música, 2018, pp. 70-80.

CAVES, R.E. Creative Industries. Contracts between Art and Commerce, Cambridge, Harvard University Press, 2002.

COULSON, S. "Collaborating in a Competitive World: Musicians' Working Lives and Understandings of Entrepreneurship", en Work, employment and Society. Vol. 26, 2012, pp. 246-261. 
DAVIS, S., GREENSTEIN, T., y GERTEISEN, M. "Effects of union type on division of household labor: Do cohabiting men really perform more housework?", en Journal of Family Issues 28, 9, 2007, pp. 1246-1272.

ELLMEIER, A. "Cultural entrepreneurialism: On the changing relationship between the arts, culture and employment”, en International Journal of Cultural Policy 9(1), 2003, pp. 3-16.

ESPASANDÍN, M. V. "Músicas y música", en DOMINZAIN, S. (coord.) Mujeres de la cultura. Escritoras, artesanas, del teatro, de la música, del cine y la televisión, Montevideo, Trilce, 2012, pp. 71-81.

ESOPE. Precarious Employment in Europe. A Comparative Study of Labor Market Related Risks in Flexible Economies. Informe final. Bruselas, European Commission, 2005.

FIM (Federación Internacional de Músicos). The Artist in Our Society. Status of Music Performers. Informe de la FIM para el congreso de la Unesco sobre el estatuto del artista, 1997.

FUWA, M. "Macro-level gender inequality and the division of household labor in 22 countries", en American Sociological Review, 69, 1, 2004, pp. 751-767.

GONZÁLEZ, S. "Primer Informe desde una perspectiva de género del sector de la música", Montevideo, MEC/OPP/ SNU, 2019.

GUIRAO, C. "La perspectiva de género en la cultura española. Mujeres y cultura en cifras" en Visiones multidisciplinares de la igualdad entre mujeres y hombres, Murcia, Laborum, 2019.

HARAWAY, D. Ciencia, cyborgs y mujeres. La reinvención de la Naturaleza, Madrid, Cátedra, 1995.

HESMONDHALGH, D., Baker, S. Creative Labour. Nueva York, Routledge, 2011.

HIMMELWEIT, S. "El descubrimiento del trabajo no remunerado: consecuencias sociales de la expansión del término trabajo" en CARRASCO, C., BORDERÍAS, T., y TORNS, T. El trabajo de cuidados. Historia, teórica y políticas. Madrid, Los libros de la catarata, 2011, pp.199-224.

HUNT, P. "The Sociology of Music", en ODELL, K. (ed.) Cambridge Handbook of Sociology: Specialty and Interdisciplinary Studies. Cambridge, Cambridge University Press, 2018, pp. 304-310.

IM, Desigualdades de género en la música. Reflexiones a partir del II Simposio Internacional de Mujeres Directoras de Orquesta. Montevideo, IM, 2019.

IM-RDM, Las mujeres DJ en la música uruguaya. Montevideo, Intendencia de Montevideo, 2018, pp. 60. 
INFANTINO, J. “Trabajar como artista. Estrategias, prácticas y representaciones del trabajo artístico entre jóvenes artistas circenses”, en Cuadernos de Antropología Social No 34, 2011, pp. 141-163.

INMUJERES, Estadísticas de género 2017, Montevideo, Ministerio de Desarrollo Social, 2017, pp. 42. $\quad$ Disponible online: http://repositorio.mides.gub.uy:8080/xmlui/bitstream/handle/123456789/987/eg\%202017.pdf ?sequence $=1 \&$ isAllowed $=y$

KAN, M. Y., SULLIVAN, O., y GERSHUNY, J. "Gender convergence in domestic work: discerning the efects of interactional and institutional barriers from large-scale data", en Sociology, 45, 2, 2011, pp. 234-51.

KAPLAN, A. “Invisible Work”, en Social Problems, 34, 5, 1987, pp. 403-415.

KERGOAT, D. "De la relación social de sexo al sujeto sexuado", en Revista Mexicana de Sociología, año 65, núm. 4, octubre-diciembre 2003, México, pp. 841-861.

KNUDSEN, K., y WÆRNESS, K. "National context and spouses' housework in 34 countries", en European Sociological Review, 24, 1, 2008, pp. 97-113.

LIMA, A. La participación de las mujeres en Murga Joven: ¿empoderamiento restringido? Tesis de grado, Facultad de Ciencias Sociales, UdelaR, Montevideo, 2013.

LOREY, I. "Gubernamentalidad y precarización de sí. Sobre la normalización de los productores y productoras culturales”, 2006. EIPCP (Instituto Europeo para Políticas Culturales Progresivas).

MAURO, K. "Entre el mundo del arte y el mundo del trabajo. Herramientas conceptuales para comprender la dimensión laboral del trabajo artístico", en Telón de fondo, revista de teoría y crítica teatral, Uba. núm. 27, 2018, pp.114-143.

MENGER, P. “Artistic Labor Markets and Careers, en Annual Review of Sociology, Vol. 25, 1999, pp. 541-574.

MORENO, S. "Uso del tiempo, desigualdades sociales y ciclo de vida", en Política y Sociedad, 46 (3), 2009, pp. 191-202.

MURILLO, S. El mito de la vida privada. De la entrega al tiempo libre, Madrid, Editorial Siglo XXI, 1996.

OIT. "El futuro del trabajo y de la calidad en la sociedad de la información: el sector de los medios de comunicación, la cultura y las industrias gráficas", 2004.

"Las relaciones de trabajo en las industrias de los medios de comunicación y la cultura". Ginebra, 2014.

Care work and care jobs for the future of decent work, Génova, International Labour Office, 2018. 
OLIVERA GUADARRAMA, R., ALFARO HUALDEHualde, A., y LÓPEZ ESTRADA, S. "Precariedad laboral y heterogeneidad ocupacional: una propuesta teórico-metodológica", en Revista Mexicana de Sociología 74, núm. 2, 2012, pp- 213-243.

PNUD. El futuro en foco. Cuadernos sobre Desarrollo Humano Desigualdades persistentes: mercado de trabajo, calificación y género, Montevideo, PNUD, 2014.

ROY, W., y DOWD, T. "What Is Sociological about Music", en Annual Review of Sociology, 36 (1), 2010, pp. 183-203.

RUIZ, V. "Pasado y presente en el candombe. Una mirada histórica. Relevamiento bibliográfico, documental y testimonial", en MEC Patrimonio vivo de Uruguay. Relevamiento de Candombe, Montevideo, UNESCO, 2015, pp. 7-65.

SAYER, L. "Trends in housework", en TREAS, J. y DROBNIC, S. (ed.) Dividing the domestic: Men, women and household work in cross-national perspective, Stanford, Stanford University Press, 2010.

SEVILLA-SANZ, A., GIMÉNEZ-NADAL, J. I., y FERNÁNDEZ, C. “Gender roles and the division of unpaid work in Spanish households", en Feminist Economics, 14, 1, 2010, pp.137184.

TAPIA, C. "Sobre el falso concepto de participación en educación", en CISMA Revista del Centro Telúrico de Investigaciones Teóricas, 1 (2), 2011, pp. 1-10.

TORNS, T., BORRÁS, V., MORENO, S., y RECIO, C. "Nuevas profesiones para la organización social del cuidado cotidiano", Estudios e investigaciones, Universidad Autónoma de Barcelona, 2012.

,"El trabajo y el cuidado: cuestiones teórico-metodológicas desde la perspectiva de género" en EMPIRIA Revista de Metodología de Ciencias Sociales, 15,1, 2008, pp. 53-73.

TREAS, J., y DROBNIC, S. (Eds.) Dividing the domestic: Men, women and household work in cross-national perspective, Stanford, Stanford University Press, 2010.

UNESCO. Recomendación relativa a la condición del artista. Belgrado, 1980. Recuperado: http://portal.unesco.org/es/ev.phpURL_ID=13138\&URL_DO=DO_TOPIC\&URL_SECTION $=201 . \mathrm{html}$

VARELA, M. "Los colectivos". En VARELA, M. (coord.) Apuntes 1.0. Músic@s y Colectivos. Montevideo, Fans de la Música, 2018, pp.5-17. 